\title{
ALGUNS ASPECTOS DA BIOLOGIA DE BIOMPHALARIA GLABRATA (SAY, 1818) E BIOMPHALARIA TENAGOPHILA (D'ORBIGNY, 1835) (PULMONATA, PLANORBIDAE). I — DURAÇÃO DO PERIODO EMBRIONÁRIO *
}

KAWAZOE, U. - Alguns aspectos da biologia de Biomphalaria glabrata (Say, 1818) $e$ Biomphalaria tenagophila (D'Orbigny, 1835) (Pulmonata, Planorbidae). I. Duração do periodo embrionário. Rev. Saúde públ., S. Paulo, 10:57-64, 1976 .

RESUMO: Foram realizadas observações sobre a duração do período embrionário de Biomphalaria glabrata e Biomphalaria tenagophila, durante um ano, em condições de laboratório. As médias, expressas em dias, encontradas para Biomphalaria glabrata $e$ Biomphalaria tenagophila foram respectivamente de 7,6 e 8,0 dias. Essas diferenças não foram significativas ao nivel de $5 \%$. o desenvolvimento mais rápido dos embriōes até a eclosão verificon-se na estação quente, em ambas as espécies, quando a temperatura média da água foi de aproximadamente $28^{\circ} \mathrm{C}$. Após a análise da matriz de correlação verificou-se que a influência da temperatura sobre o tempo de desenvolvimento embrionário foi pouco intensa: Biomphalaria glabrata apresentou maior correlação com a temperatura minima do ambiente $(36,2 \%)$ e com a temperatura da água $(36,0 \%)$ enquanto Biomphalaria tenagophila apresentou maior correlação com a temperatura minima do ambiente (2\%,0\%).

Unitermos: Planorbideos, biologia. Biomphalaria glabrata. Biomphalaria tenagophila. Esquistossomose mansônica.

A biologia dos moluscos do gênero glabrata. Posteriormente, Rey ${ }^{18}$ (1956) Biomphalaria tem sido estudada por di- publicou monografia visando especialmenversos autores, principalmente no que se te esta espécie, com algumas observações refere a espécie Biomphalaria glabrata, sobre a espécie $B$. tenagophila. Outro um dos hospedeiros intermediários mais trabalho importante sobre a biologia da importantes do Schistosoma mansoni, no B. glabrata foi feito por Perlowagora ${ }^{12}$ Brasil.

Brumpt $^{4}$ (1941) foi um dos primeiros pesquisadores a estudar a biologia da $B$. (1958).

Por outro lado, B. tenagophila apresenta no Estado de São Paulo grande impor-

* Trabalho realizado, em parte, com o auxilio da FAPESP, CNPq e CAPES, em diferentes etapas da pesquisa.

* Do Setor de Parasitologia da Universidade de Campinas (UNICAMP). Caixa Postal 1170 - Campinas, SP - Brasil. 
KAWAZOE, U. - Alguns aspectos da biologia de Biomphalaria glabrata (Say, 1818) e Biomphalaria tenagophila (D'Orbigny, 1835) (Pulmonata, Planorbidae). I. Duração do periodo embrionário. Rev. Saude públ., S. Paulo, 10:57-64, 1976.

tância epidemiológica devido a sua comprovada suscetibilidade à infecção pela cepa local de $S$. mansoni. Os trabalhos de Piza e cols. ${ }^{14,15}$ (1960) e Ramos e cols. ${ }^{16,17}$ (1961, 1969) tem confirmado o importante papel da B. tenagophila na manutenção de focos de esquistossomose, principalmente nas regióes do Vale do Paraíba, Vale do Ribeira e Baixada Santista. Recentemente foram verificados vários focos isolados de esquistossomose na região de Campinas por Magalhães e cols. $^{s}$ (1973).

No entanto, face a importância dessa espécie, sua biologia não tem sido devidamente estudada, embora a espécie esteja se tornando importante vetor da esquistossomose. Somente Rey ${ }^{18}$ (1956) e Magalhães e cols. 5, 6, 7 (1968, 1969, 1971) estudaram alguns aspectos da biologia, em observações realizadas em curto espaço de tempo.

Deste modo, propomos o estudo de alguns aspectos da biologia desses planorbídeos, em laboratório, comparando as duas espécies já mencionadas, isto é, $B$. glabrata cuja biologia é bastante conhecida e $B$. tenagophila, cuja espécie não se acha bem estudada.

Neste trabalho apresentamos os resultados de um dos parâmetros que se refere à duração do período embrionário.

\section{2 - MATERIal E métodos}

Foi realizado estudo biológico comparado entre exemplares de duas populações de planorbídeos: $B$. glabrata procedente de Salvador (Bahia) e B. tenagophila de Campinas (São Paulo).

$O$ trabalho foi executado em um ano, em condições de laboratório, a fim de que fossem verificadas possíveis variações sazonais. As observações foram agrupadas em períodos de 20 dias.

Em cada período foram utilizados 12 pares de caramujos de cada espécie cujo diâmetro máximo estava compreendido entre 8 a $11 \mathrm{~mm}$. Os moluscos foram colocados aos pares a fim de se obter reprodução cruzada. Os pares de caramujos foram depositados em "beckeres" de $600 \mathrm{ml}$, contendo água não clorada com 300 ppm de carbonato de cálcio suficiente para haver saturação (Azevedo ${ }^{\mathbf{1}}$, 1967).

Para oviposição dos moluscos foi colocada, em cada recipiente, uma lâmina quadrada de plástico com $4 \mathrm{~cm}$ de lado (Olivier e cols. ${ }^{9}, 1962$ ).

Tendo por finalidade facilitar a observação das desovas, os frascos não continham substrato arenoso nem plantas aquáticas.

A água dos frascos era renovada semanalmente para que fosse evitada a multiplicação excessiva de algas e o acúmulo de detritos.

A alimentação foi feita através da deposição de folhas de alface nos frascus, em quantidade suficiente de modo que fossem totalmente consumidas pelos planorbícieos em 24 horas. Dessa maneira evitou "se a oviposição na superfície do alimento.

O desenvolvimento dos embriōes foi observado desde a deposição dos ovos até a data correspondente ao primeiro dia da eclosão dos caramujos da cápsula ovífera. Este período foi expresso em dias.

Foram registradas as temperaturas máxima e mínima do ambiente e a temperatura da água bem como a pressão atmosférica, em cada período da experiência.

$$
3 \text { - RESULTADOS }
$$

B. glabrata apresentou média de $7,6 \pm$ 1,38 dias para a duração do periodo embrionário enquanto que os exemplares $B$. tenagophila apresentaram média de $8,0 \pm 1,54$ dias (Tabela 1 ).

A análise da variância (teste F) mostrou diferença significativa entre as médias, expressas em dias, dos desenvolvimentos embrionários de cada período de observação de $B$. glabrata. 0 mesmo teste 
KAWAZOE, U. - Alguns aspectos da biologia de Biomphalaria glabrata (Say, 1818) e Biomphalaria tenagophila (D'Orbigny, 1835) (Pulmonata, Planorbidae). I. Duração do periodo embrionário. Rev. Saúde públ., S. Paulo, 10:57-64, 1976.

TABELA 1

Duração média, em dias, do periodo embrionário de $B$. glabrata e $B$. tenagophila

\begin{tabular}{|c|c|c|c|c|c|c|}
\hline \multirow{2}{*}{ Perfodos de observação } & \multicolumn{3}{|c|}{ B. glabrata } & \multicolumn{3}{|c|}{ B. tenagophila } \\
\hline & $n^{*}$ & $\bar{x}$ & $\mathbf{s}$ & $n^{*}$ & $\vec{x}$ & $\mathbf{s}$ \\
\hline \multicolumn{7}{|l|}{1.0} \\
\hline$(13-03-69$ a $03-04-69)$ & 48 & 7,3 & 1,02 & 53 & 7,6 & 0,74 \\
\hline \multicolumn{7}{|l|}{2.0} \\
\hline$(15-04-69$ a $19-05-69)$ & 50 & 7,5 & 0,99 & 50 & 7,4 & 0,99 \\
\hline \multicolumn{7}{|l|}{3.0} \\
\hline$(22-05-69$ a 23-06-69) & 50 & 8,5 & 1,66 & 55 & 8,9 & 1,63 \\
\hline \multicolumn{7}{|l|}{$4.0^{\circ}$} \\
\hline$(26-06-69$ a $30-07-69)$ & 50 & 7,8 & 1,54 & 50 & 6,8 & 1,39 \\
\hline \multicolumn{7}{|l|}{5.0} \\
\hline$(07-08-69$ a $09-09-69)$ & 50 & 7,8 & 1,35 & 51 & 8,2 & 1,11 \\
\hline \multicolumn{7}{|l|}{$6.0^{\circ}$} \\
\hline$(13-09-69$ a $16-10-69)$ & 143 & 7,9 & 1,44 & 121 & 8,3 & 1,48 \\
\hline \multicolumn{7}{|l|}{7.0} \\
\hline$(16-10-69$ a $17-11-69)$ & 190 & 7,7 & 1,64 & 121 & 8,9 & 1,67 \\
\hline \multicolumn{7}{|l|}{8.0} \\
\hline$(17-11-69$ a $22-12-69)$ & 49 & 7,9 & 1,25 & 63 & 11,4 & 3,80 \\
\hline \multicolumn{7}{|l|}{9.0} \\
\hline$(24-12-69$ a $20-01-70)$ & 69 & 7,3 & 1,45 & 110 & 7,5 & 1,35 \\
\hline \multicolumn{7}{|l|}{10.0} \\
\hline$(28-01-70$ a 26-02-70) & 110 & 7,0 & 1,16 & 98 & 6,8 & 1,07 \\
\hline \multicolumn{7}{|l|}{11.0} \\
\hline$(28-02-70$ a $30-03-70)$ & 116 & 7,1 & 1,71 & 89 & 6,5 & 1,71 \\
\hline Total & 925 & - & 一 & 861 & $一$ & 一 \\
\hline Média geral & 一 & 7,6 & 1.38 & - & 8,0 & 1,39 \\
\hline
\end{tabular}

* Número total de cápsulas oviferas observados.

foi aplicado a $B$. tenagophila observandose resultado semelhante.

A análise estatística da diferença da média entre as duas populações não mostrou significância ao nível de $5 \%$. Por outro lado, foi calculada a matriz de correlação entre os períodos embrionários de B. glabrata e B. tenagophila e os fato- res ambientes (temperatura máxima, mínima, temperatura da água e pressão atmosférica).

$O$ resultado revelou que os coeficientes de correlação foram significativamente diferentes de zero, havendo certa dependência entre os fatores acima mencionados 
KAWAZOE, LT. - Alguns aspectos da biologia de Biomphalaria glabrata (Say. 1818) e Biomphalaria tenagophlla (D'Orbigny, 1835) (Pulmonata, Planorbidae). I. Duração do periodo embrionário. R\&r. Salude públ., S. Paulo, 10:57-64, 1976.

e a duraçāo do período embrionário de B. glabrata.

O periodo embrionário de $B$. tenagophila apresentou coeficiente de correlação significativamente diferente de zero em relação à temperatura máxima e a da aqua (Tahela 21.

TABELA 2

Coeficiente de correlacão entre duração do periodo embrionário de $B$. glabrata e $B$. tenagophlla e fatores ambientes

\begin{tabular}{l|l|l|}
\hline Fatores ambientes & B. glabrata & B. tenagophila \\
\hline
\end{tabular}

Temp. máxıma

Temp. minima médla

Temp. média da àgua

P. atmosférica média

$\begin{array}{rr}-0.149 & -0.269 \\ -0.362 & -0.055 \\ -0.360 & -0.158 \\ 0,228 & 0,005\end{array}$

Os dados referentes ao registro dos fatores ambientes e da água encontram-se na Tabela 3 .

A Figura mostra os dados comparados entre B. glabrata e B. tenagophila rela- cionados com a temperatura máxima, mínima e da água.

\section{4 - COMENTários E CONCLUSós}

Ohserva-se na Tabela 1 que as espécies $B$. glabrata e $B$. tenagophila nāo apresentaram diferença significativa. em relação à média da duração do período embrionário. Esse fato foi verificado também por Rey " 1956) entre as duas espécies mencionadas. sugerindo comportamentos equivalentes. No entanto. Magalhäes e cols.:- (1968. 1971). além de terem encontrado valores mais altos para o desenvolvimento embrionário. notaram diferença significativa na duração do desenvolvimento embrionário entre as duas espécies. Entretanto, essas observações realizadas em curto espaço de tempo. com amostras pequenas. não conduzem a resultados que possam ser comparados com trahalhos realizados num espaço de tempo mais prolongado.

Analisando-se cada espécje separadamente. na Tabela 1. ohserva-se a existência de diferença significativa entre os períodos da experiência (Figura). () tempo médio mais longo da duração do

T A B E I. A 3

Temperatura do ar, pressão atmosférica e temperatura da água. registrados durante () trabalho (1969-1970)

\begin{tabular}{c|ccc|c} 
Periodos de \\
observacós
\end{tabular}


KAWAZOE, U. - Alguns aspectos da biologia de Biomphalaria glabrata (Say, 1818) e Biomphalaria tenagophila (D’Orbigny, 1835) (Pulmonata, Planorbidae). I. Duracão do periodo embrionário. Rev. Saúde públ., S. Paulo, 10:57-64, 1976.

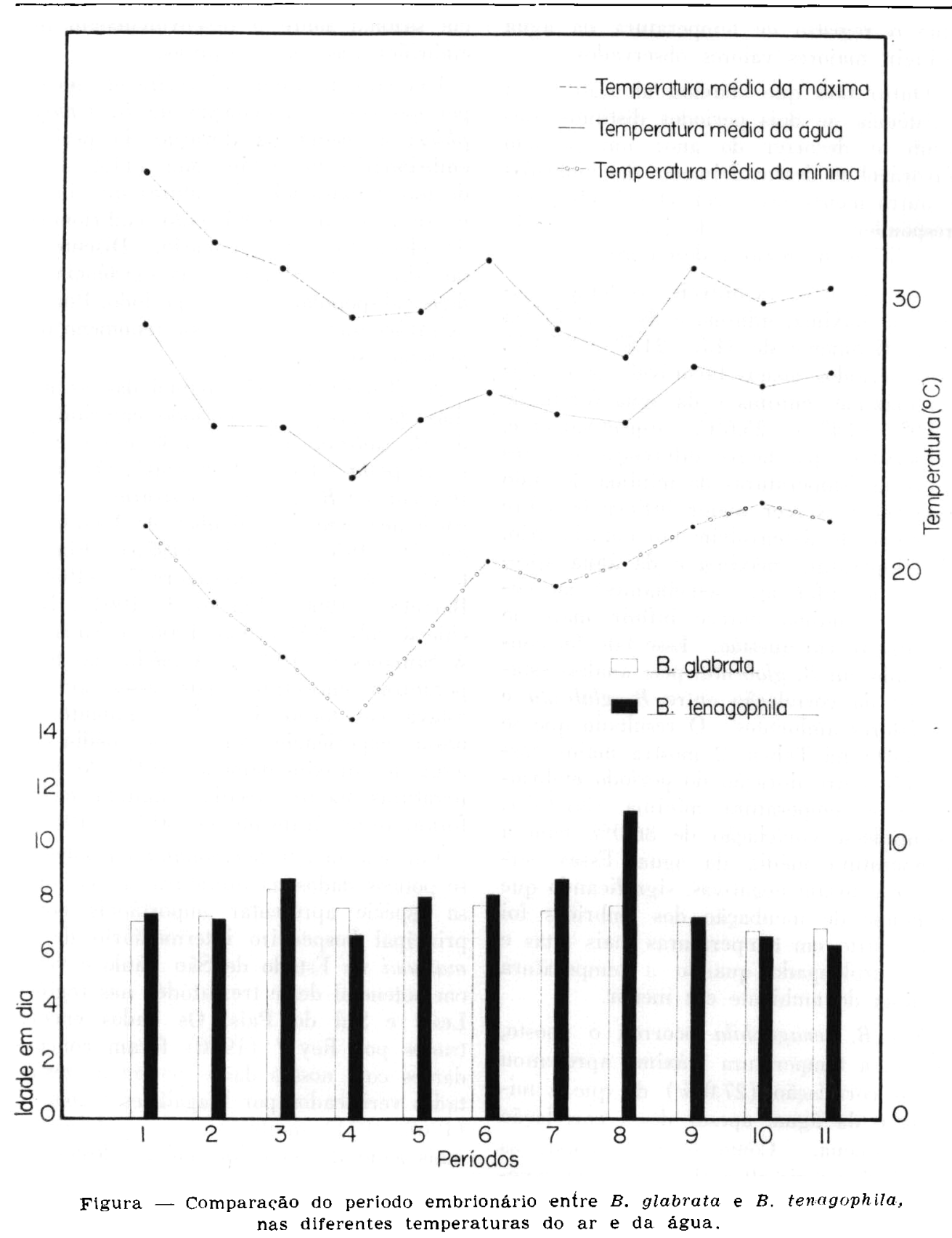

período embrionário verificou-se no $30^{\circ}$ giram os valores mais baixos. Por outro período com a média de 8.5 dias para lado, no $100^{\circ}$ e $110^{\circ}$ períodos foram veri$B$. glabrata e 8,9 dias para $B$. tenagophila. ficados os menores tempos de desenvolviEsse periodo correspondeu aos meses de mento embrionário, correspondendo aos maio-junho. onde as temperaturas atin- meses de fevereiro e março, períodos em 
KAWAZOE, U. - Alguns aspectos da biologia de Biomphalaria glabrata (Say, 1818) e Biomphalaria tenagophila (D'Orbigny, 1835) (Pulmonata, Planorbidae). I. Duraç̃o do periodo embrionárlo. Rev. Saúde públ., S. Paulo, 10:57-64, 1976.

que o registro de temperatura da água atingiu maiores valores observados.

Outro fato que chamou atenção foi a existência de dois períodos distintos, durante o decorrer do ano: um período favorável ao desenvolvimento dos enbrióes e outro menos favorável. 0 primeiro correspondeu aos meses de janeiro a maio e o último de maio a dezembro.

Nos períodos favoráveis, as temperaturas da máxima, mínima e da água foram respectivamente de $31,8^{\circ}, 21,5^{\circ}$ e $27,4^{\circ} \mathrm{C}$. Nos períodos menos favoráveis as médias da máxima, mínima e da água foram de $29,9^{\circ}, 18,4^{\circ}$ e $25,6^{\circ} \mathrm{C}$, respectivamente. Observa-se que houve diferenças maiores entre as temperaturas da mínima devendo este fator exercer maior influência sobre o tempo de desenvolvimento embrionário. A temperatura máxima e da água apresentaram diferenças semelhantes; no entanto, a última parece influir mais no parâmetro em questão. Esse fato foi confirmado em $B$. glabrata, pela análise estatística da correlação entre $B$. glabrata e os fatores ambientes. $O$ resultado que se encontra na Tabela 2 mostra maior correlação entre duração do período embrionário e temperatura mínima $(36,2 \%)$, seguindo-se correlação de $36,0 \%$ com a temperatura média da água. Essas correlações foram negativas, significando que o tempo de incubação dos embriōes foi mais curto em temperaturas mais altas $e$ mais prolongado quando a temperatura mínima do ambiente era menor.

Em B. tenagophila ocorreu o oposto, isto é, a temperatura máxima apresentou maior correlação $(27,0 \%)$ do que a mínima e da água, apesar dessa correlação ser pequena. Como se pode notar, as correlações mais altas observadas na experiência não foram muito acentuadas. Portanto as temperaturas influíram pouco no desenvolvimento embrionário desses planorbideos.

No entanto, Rey ${ }^{18}$ (1956) e Perlowagora ${ }^{12}$ (1958) verificaram nítida influên- cia sazonal sobre o desenvolvimento dos embriōes, nas suas pesquisas.

Um fato incomum foi verificado no $8 .^{\circ}$ período com a população de $B$. tenagophila: a média da duração do período embrionário foi muito alta $(11,4$ dias $)$ devido a existência de fatores que interferiram no desenvolvimento embrionário de elementos da população. Discute-se, também, a possibilidade da existência de duas sub-populações nesse período. Porém, as razões que causaram esse fenômeno não puderam ser esclarecidas.

$\mathrm{Na}$ T'abela 1, estão assinaladas as médias da duração do período embrionário de $B$. glabrata $(7,6 \pm 1,38$ dias $)$ e $B$. tenagophila (8,0 $\pm 1,39$ dias). Os dados referentes a $B$. glabrata mostraram-se concordantes com os trabalhos de Penido \& cols. ${ }^{11}$ (1951), Rey ${ }^{18}$ (1956), Pimentel $^{13}$ (1957), Perlowagora ${ }^{12}$ (1958), Barreto $^{3}$ (1960), Barbosa ${ }^{2}$ (1961), Ritchie \& cols. ${ }^{19,20}(1963,1966)$ e Sturrock \& Sturrock ${ }^{21}$ (1972). A média das temperaturas encontrada por esses autores estava em torno de $25^{\circ} \mathrm{C}$, enquanto na nossa experiência obtivemos média da água de aproximadamente $26^{\circ} \mathrm{C}$. As temperaturas máxima média e mínima média foram respectivamente de $30,7^{\circ}$ e $19,8^{\circ} \mathrm{C}$.

Em relação a $B$. tenagophila, encontramse poucos dados na literatura, apesar dessa espécie apresentar importância como principal hospedeiro intermediário de $S$. mansoni no Estado de São Paulo e vetor em potencial desse trematódeo nas regiões Leste e Sul do País. Os dados encontrados por Rey ${ }^{18}$ (1956) foram concordantes com nossos dados porém os resultados verificados por Magalhães e cols., 7 (1968, 1971) mostraram desenvolvimento mais lento dos embrióes até a eclosão.

Apesar dos nossos dados terem revelado influência pouco intensa das temperaturas, sabe-se que este é o principal fator que modifica o período de desenvolvimento embrionário como verificaram Sturrock \& Sturrock $^{21}$ (1972) que conseguiram a duração de 6 dias para o desenvolvimento 
KAWAZOE, U. - Alguns aspectos da biologia de Biomphalaria glabrata (Say, 1818) e Biomphalaria tenagophila (D'Orbigny, 1835) (Pulmonata, Planorbidae). I. Duração do período embrionário. Rev. Saúde públ., S. Paulo, 10:57-64, 1976.

dos embriōes à temperatura de $30^{\circ} \mathrm{C}$, enquanto a $25^{\circ} \mathrm{C}$ tinham obtido duração de 8 dias e a $20^{\circ} \mathrm{C}, 13$ a 18 dias.

Penido \& cols. ${ }^{11}$ (1951) obtiveram média de 5,3 dias para $B$. straminea vindos do campo e em $B$. glabrata e $B$. straminea criados no laboratório, encontraram periodo entre 2 a 8 dias. Este último resultado, porém, é duvidoso pois julga-se que 2 dias não seriam suficientes para o desenvolvimento e eclosão dos emergentes.

Tendo em vista os resultados contidos na Tabela 1 , ficou comprovado que o tempo de desenvolvimento embrionário sofre variaçôes, mesmo em planorbídeos provenientes de um mesmo local. $O$ fator que mais contribui para esta variação parece ter sido a temperatura, sendo que a temperatura mais favorável ficou compreendida entre $25^{\circ}$ a $30^{\circ} \mathrm{C}$.

\section{AGR A DECIMENTOS}

Ao Dr. Luiz Augusto Magalhães pela orientação do trabalho e ao Prof. José Ferreira de Carvalho pela análise estatística dos dados.

RSPU-B/299

KAWAZOE, U. - [Some angles of the biology of Biomphalaria glabrata (Say. 1818) and Biomphalaria tenagophila (D'Orbigny, 1835) (Pulmonata, Planorbidae). I. Duration of the embryo period]. Rev. Saúde públ., S. Paulo, $10: 57-64,1976$.

SUMmaRx: The incubation periods of Biomphalaria glabrata and Biomphalaria tenagophila were compared during one year, in the laboratory. The average time for eggs hatching was 7,6 and 8,0 days for Biomphalaria glabrata and Biomphalaria tenagophila, respectively; the difference was not significant at the 5\% level. The fastest development of the eggs was verified in the summer when the average water temperature was approximately $28^{\circ} \mathrm{C}$. The analysis of the correlation coeficient showed that the influence of temperature on the egg development period was not strong: Biomphalaria glabrata showed best correlation with minimum air temperature $(36,2 \%)$ and with water temperature $(36,0 \%)$, while Biomphalaria tenagophila correlated best with minimum air temperature $(2 \%, 0 \%)$.

Uniterms: Planorbidae biology. Biomphalaria glabrata. Biomphalaria tenagophila. Schistosomiasis mansoni.

\section{REFERENCIAS BIBLIOGRAFICAS}

1. AZEvedo, J. F. et al. - Calcium absorption by Australorbis glabratus and Physa acuta in constant concentration environment. Rev. Inst. Med. trop. S. Paulo, 9:419-28, 1967.

2. BARBOSA, F. S. - História natural dos planorbídeos transmissores de Schistosoma mansoni no Nordeste do Brasil. In: Simpósio sobre Bioquimica de Planorbideos, Curitiba, 1961. p. 11-28.
2 BARRETO, A. C. - Esquietromose mansônica na cidade de salvador. Estudo do vetor, relações parasito hospedeiro e aspectos epidemiológicos. Bol. Fund. Gonçalo Moniz, 16:1-80, 1960.

4. BRUMPT, E. - Observations biologiques diverses concernant Planorbis (Australorbis) glabratus hote intermédiaire de Schistosoma mansoni. Ann. Parasitol., 18:9-45, 1941. 
KAWAZOE, U. - Alguns aspectos da biologia de Biomphalaria glabrata (Say, 1818) e Biomphalaria tenagophila (D'Orbigny, 1835) (Pulmonata, Planorbidae). I. Duração do periodo embrionário. Rev. Salide públ., S. Paulo, 10:57-64, 1976.

5. MAGALHÃS, L. A, et al. - Estudo da dinâmica populacional de Biomphalaria glabrata e $B$. tenagophila. I. Estudo comparativo da postura e do desenvolvimento das desovas de populações de Biomphalaria glabrata e $B$. tenagophila. Nota Prévia. Rev. paul. Med., 72:268-9, 1968.

6. MAGAlHãES, L. A. \& CARVALHO, J. F. - Estudo da postura de duas populaçoes de planorbídeos. Rev. Soc. bras. Med. trop., 3:245-7, 1969.

7. MAGAlHAES, L. A. \& DE LLCCA, O. Determinação do periodo de desenvolvimento e da viabilidade das desovas de duas populações de Biomphalaria glabrata e Biomphalaria tenagophila (Mollusca, Planorbidae). Rev. Soc. bras. Med. trop., 5:307-13, 1971

8. MAGALHAES, L. A. et al. - Aspectos epidemiológicos da esquistossomose mansônica na Região da Represa de Americana, Estado de São Paulo, Brasil. Rev. Saude públ., S. Paulo, 7:21-2, 1973.

9. OLIVIER, L. et al, - The action of very low concentrations of sodium pentrachlorophenate on fresh]y laid eggs of Australorbis glabratus. Bull. Wld. Hlth. Org., 27:87-94, 1962.

10. PARAENSE, W. L. - Planorbídeos hospedeiros intermediários do Schistosoma mansoni. In: CLNHA, A. S. daEsquistossomose manson. São Paulo, Ed. Universidade de São Paulo, 1970. p. $13-30$.

11. PENIDO, H. M. et al. - Observações sobre as posturas e o tempo de evolução de duas espécies de caramujos encontrados no Vale do Rio Doce. Rev. Serv. Saúde públ., Rio de Janeiro, 4:407-12, 1951.

12. PERLOWAGORA, S. A. - Studies on the biology of Australorbis blabratus, schistosoma bearing brasilian snail. Rev. bras. Malar, 10:459-529, 1958 .
13. PINENTEL. D. - Life history of $A u s$ tralorbis glabratus the intermediate snail host of Schistosoma mansoni in Puerto Rico. Ecology, 38:576-80, 1957.

14. PIZA, J. T. \& RAMOS, A. S. - Os focos autóctones de esquistossomose no Estado de São Paulo. Arq. Hig. S. Paulo, 25:261-71. 1960.

15. PIZA, J. T. et al. - Vale do Paraíba. Foco endèmico de esquistossomose. Arq. Hig. S. Paulo, 25:35-40, 1960.

16. RAMOS, A s. et al. - Observacōes sobre Australorbis tenagophilus, transmissor da esquistossomose mansônica. Arq. Hig. S. Paulo, 26:121-4, 1961.

17. RAMOS, A. S. et al. - Focos ativos de esquistossomose mansoni no Vale do Ribeira, Estado de São Paulo, Brasil. Rev. Saúde pübl., S. Paulo, 3: 59-65, 1969.

18. REY, L. - Contrbuicáo para o comportamento da morfologia, biologia e ecologia dos planorbídeos brasileiros transmissores da esquistossomose. Rio de Janeiro, Servico Nacio. nal Educação Sanitária, 1966.

19. RITCHIE, L. S. et al. - Biological potentials of Australorbis glabratus: growth and maturation. J. trop. Med. Hyg., 12:264-8, 1963.

20. RITCHIE, L. S. et al. - Biological potentials of Australorbis glabratus. Life span and reproduction. Amer. J. trop. Med. Hyg., 15:614-7, 1966.

21. STURROCK, R. F. \& STURROCK, B. M. - The influence of temperature on the biology of Biomphalaria glabrata (Say), intermediate host of Schistosoma mansoni on St. Lucia. West Indies. Ann. trop. Med. Parasit., 66: 385-90, 1972 .

Recebilo para publicaşo em 15/09/1975 A provado para publicasio em 05/01/19r6 